\title{
Surface-Engineered Nanostructure-Based Efficient Nonpolar GaN Ultraviolet Photodetectors
}

\author{
Monu Mishra, ${ }^{\dagger \dagger}$ Abhiram Gundimeda, ${ }^{\dagger \neq}$ Shibin Krishna, ${ }^{\dagger, \ddagger}$ Neha Aggarwal, ${ }^{\dagger,}$ Lalit Goswami, ${ }^{\ddagger}$ \\ Bhasker Gahtori, ${ }^{\ddagger}$ Biplab Bhattacharyya, ${ }^{\dagger, \S}$ Sudhir Husale, ${ }^{\S}$ and Govind Gupta*, ${ }^{\dagger, \ddagger}$, \\ ${ }^{\dagger}$ Academy of Scientific and Innovative Research, CSIR-NPL Campus, Dr. K.S. Krishnan Marg, New Delhi 110012, India \\ ${ }^{\ddagger}$ Advanced Materials and Devices Division and ${ }^{\S}$ Time and Frequency, Electrical \& Electronics Metrology Division, CSIR-National \\ Physical Laboratory, Dr. K.S. Krishnan Marg, New Delhi 110012, India
}

\section{Supporting Information}

\begin{abstract}
Surface-engineered nanostructured nonpolar $(11 \overline{2} 0)$ gallium nitride $(\mathrm{GaN})$-based high-performance ultraviolet (UV) photodetectors (PDs) have been fabricated. The surface morphology of a nonpolar GaN film was modified from pyramidal shape to flat and trigonal nanorods displaying facets along different crystallographic planes. We report the ease of enhancing the photocurrent (5.5-fold) and responsivity (6fold) of the PDs using a simple and convenient wet chemicaletching-induced surface engineering. The fabricated metalsemiconductor-metal structure-based surface-engineered UV PD exhibited a significant increment in detectivity, that is, from 0.43 to $2.83\left(\times 10^{8}\right)$ Jones, and showed a very low noiseequivalent power $\left(\sim 10^{-10} \mathrm{~W} \mathrm{~Hz}^{-1 / 2}\right)$. The reliability of the
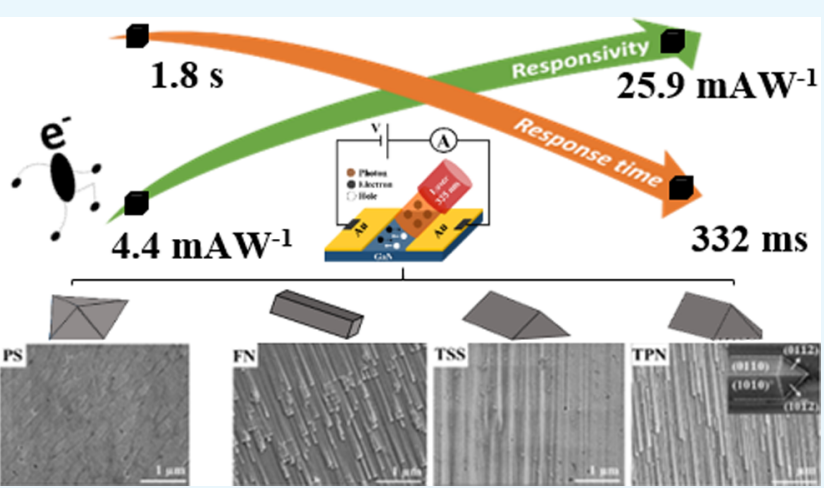
nanostructured PD was ensured via fast switching with a response and decay time of 332 and $995 \mathrm{~ms}$, which were more than five times faster with respect to the unetched pyramidal structure-based UV PD. The improvement in device performance was attributed to increased light absorption, efficient transport of photogenerated carriers, and enhancement in conduction cross section via elimination of recombination/trap centers related to defect states. Thus, the proposed method could be a promising approach to enhance the performance of GaN-based PD technology.
\end{abstract}

\section{INTRODUCTION}

Ultraviolet (UV) photodetectors (PDs) have drawn researchers' interest because of their dynamic applications in rising global concerns such as environmental pollution monitoring, cancer detection, flame alarms, future memory storage, water purification, binary switches in imaging techniques, space-tospace communication, and next-generation defense warfare technology. ${ }^{1,2}$ The three spectral regions in the UV range are UV-A (400-320 nm), UV-B (320-280 nm), and UV-C (280$200 \mathrm{~nm})$, in which UV-B and UV-C rays with shorter wavelength and higher energies are absorbed by the stratospheric ozone layer, whereas UV-A with long wavelength passes through the atmosphere onto the earth's surface. ${ }^{3}$ Considering the importance of monitoring UV-A radiation, a highly responsive and fast photodetective device is essential.

Commercially available narrow band gap ( $\mathrm{Si}, \mathrm{GaAsP}, \mathrm{GaP}$, etc.)-based PDs possess high noise and leakage currents, low operating temperature, requirement of optical filters, etc., which hinder their integration in wearable technologies. ${ }^{4}$ On the other hand, wide band gap (WBG) semiconductors, being the ideal candidate for the development of efficient UV PDs, can easily overcome the aforementioned challenges. ${ }^{5}$ Among these, gallium nitride $(\mathrm{GaN})$ with direct WBG $(3.4 \mathrm{eV})$ and superior material properties such as high saturation velocity, high chemical stability, high carrier mobility, high temperature, and frequency operation has proven its potential for employment in UV photodetection under harsh environmental conditions. ${ }^{6}$ GaN films grown along different directions (polar or semipolar) exhibit polarization effect and variation in chemical structure and electronic properties. ${ }^{7-9}$ The presence of polarization-induced Stark effect in polar GaN films (c-plane) significantly affects the carrier transport and radiative recombination, ${ }^{10}$ which initiated research on exploring nonpolar GaN films for device fabrication. ${ }^{11-13}$ Nonpolar GaN structures enhance the performance of the device via elimination of polarization-induced electric field and quantum-confined Stark effect which may impede the device performance. $^{8}$ To address the polarization effect, Mukundan et al. fabricated polar and nonpolar GaN-based UV PDs, where nonpolar GaN-based UV PDs produced enhanced responsivity and fast response time than polar GaN UV PDs. ${ }^{12}$ Recently,

Received: December 20, 2017

Accepted: February 14, 2018

Published: February 26, 2018 


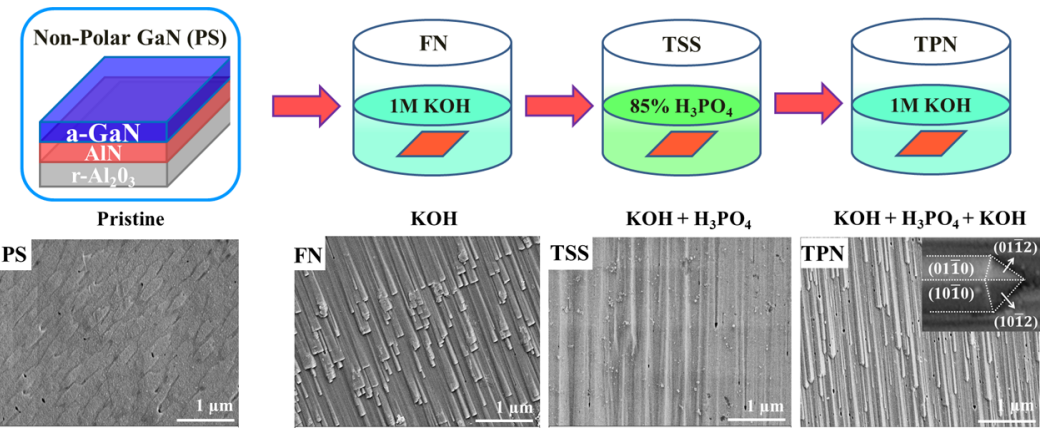

Figure 1. FESEM images displaying sample morphology after different stages of etching: PS, FN, TSS, and TPN GaN films.

Gundimeda et al. also fabricated nonpolar GaN-based UV PDs and reported enhanced responsivity. ${ }^{13}$

Nanostructured devices offer quantum-, surface-, dielectric-, and optical-confinement effects, which lead to higher light absorption, increased photoconductive gain, enhanced responsivity, and faster switching. ${ }^{14,15}$ The high surface-to-volume (i.e., aspect) ratio of a nanostructured/textured film encourages scattering of incident photons, reduces reflection losses, and increases the absorption of incident radiation which ultimately contribute in enhancing the device efficiency via direct or indirect mode. Besides, the low-dimensional conductive channel of nanostructures could confine the active area of charge carriers and shorten the carrier transit time. ${ }^{16-18}$ Theoretical reports ${ }^{19,20}$ as well as experimental findings ${ }^{21}$ reveal effective light trapping and significant improvement in device performance using nanostructured/textured surfaces.

A nanostructured surface can be obtained either by the direct growth of nanostructures, that is, by chemical vapor deposition, physical vapor deposition (PVD) including sputtering/evaporation/epitaxial growth method, etc., or via engineering the flat surfaces to develop nanostructures using wet/dry etching processes. ${ }^{22-26}$ Kumar et al. ${ }^{27}$ proposed the growth of InN- and GaN-based self-assembled flower-shaped nanostructures via molecular beam epitaxy (MBE), while Aggarwal et al. ${ }^{28}$ reported nanoflower decoration on $\mathrm{GaN}$ surfaces to enhance light absorption in the fabricated UV PDs. However, compared to growth-related challenges, surface engineering is a simple and convenient approach to develop ordered nanostructures. Owing to its high synthesis versatility, negligible surface damage, absence of hydrogen contaminants, higher selectivity, simple execution, and low cost, wet chemical etching is preferred over other techniques for the fabrication of $\mathrm{GaN}$ micro- and nanostructures, which offers a huge potential for detection applications. ${ }^{29}$ However, challenges regarding the impact of wet chemically etched nanostructures on PD applications are yet to be explored.

In the present article, we report chemically modified (using hot $\mathrm{KOH}$ and $\mathrm{H}_{3} \mathrm{PO}_{4}$ solution) surface-engineered nonpolar GaN PDs fabricated using the metal-semiconductor-metal (MSM) approach. Photodetection studies were performed to analyze the responsivity, detectivity, noise-equivalent power (NEP), rise time, and decay time of the fabricated UV PDs. The highly ordered nanostructure-based UV PDs displayed significant enhancement in device performance (compared with the pristine film), ascertaining their potential in the development of highly efficient GaN-based optoelectronic devices.

\section{RESULTS AND DISCUSSION}

The morphological transformations of the surface-engineered GaN film investigated via field emission scanning electron microscopy (FESEM) analysis are represented in Figure 1. The unetched $\mathrm{GaN}$ film displayed pitted morphology with uniformly distributed pyramidal-shaped faceted structures (average size $\approx 400 \pm 50 \mathrm{~nm}$ ) over the surface. The etching of pyramidal structures (PS) in hot $\mathrm{KOH}$ solution transformed the PS into flat nanorods (FN) oriented toward the (11102) direction, with their width varying between 60 and $300 \mathrm{~nm}$. Further, $\mathrm{FN}$ were immersed in $\mathrm{H}_{3} \mathrm{PO}_{4}$ solution which resulted in the development of trigonal-shaped structures (TSS) along with a slight broadening of the surface pits. The observed two facets of TSS were ascribed to the $(01 \overline{1} 0)$ and $(10 \overline{1} 0)$ planes of the hexagonal GaN. Finally, trigonal prism-shaped nanorods (TPN) with triangular termination were developed when TSS underwent $\mathrm{KOH}$ etching. The width of the nanorods was observed to be in between 70 and $320 \mathrm{~nm}$. The side facets of the TPN consisted of the same $(01 \overline{1} 0)$ and $(10 \overline{1} 0)$ planes, whereas the triangular termination was attributed to the (01) and $(10 \overline{1} 2)$ planes of the GaN wurtzite crystal structure (as shown in the inset of Figure 1).

The existence of pentagonal pits was evident throughout the etching treatment, though a slight variation in the pit size was observed. The root-mean-square (rms) roughnesses of the PS, FN, TSS, and TPN films were examined by atomic force microscopy (AFM) measurements (Figure S1 of the Supporting Information) and were obtained as 3.9, 6.0, 6.8, and 29.0 $( \pm 0.2) \mathrm{nm}$, respectively. The increment in the rms roughness indicates higher surface-to-volume ratio of the surfaceengineered nanostructures, which is expected to increase the charge generation, eliminate the surface trap states to minimize surface recombination, and ultimately improve the photoconductivity. Moreover, because of the formation of the network structure, the surface of the PD would have larger contact area, which results in enhanced carrier collection and faster response time. ${ }^{30}$ Jung et al. ${ }^{31}$ reported that the etching rate/mechanism in $\mathrm{GaN}$ has a direct correlation with the surface chemical states. The GaN film in an alkaline medium reacts with water, leading to the formation of gallium oxide which gets dissolved in the solution. ${ }^{31}$ The low activation energy of the chemical reaction in the case of semipolar/ nonpolar $\mathrm{GaN}$ films plays a critical role in governing the etching rate of the reaction. The presence of negatively charged hydroxyl $\left(\mathrm{OH}^{-}\right)$ions on the surface of nonpolar $\mathrm{GaN}$ films promotes chemical etching and assists in faceting/texturing of the surface along various crystallographic planes with higher chemical stability. ${ }^{32-34}$ Further, the broadening of the pentagonal-shaped surface pits in TSS and TPN can be 

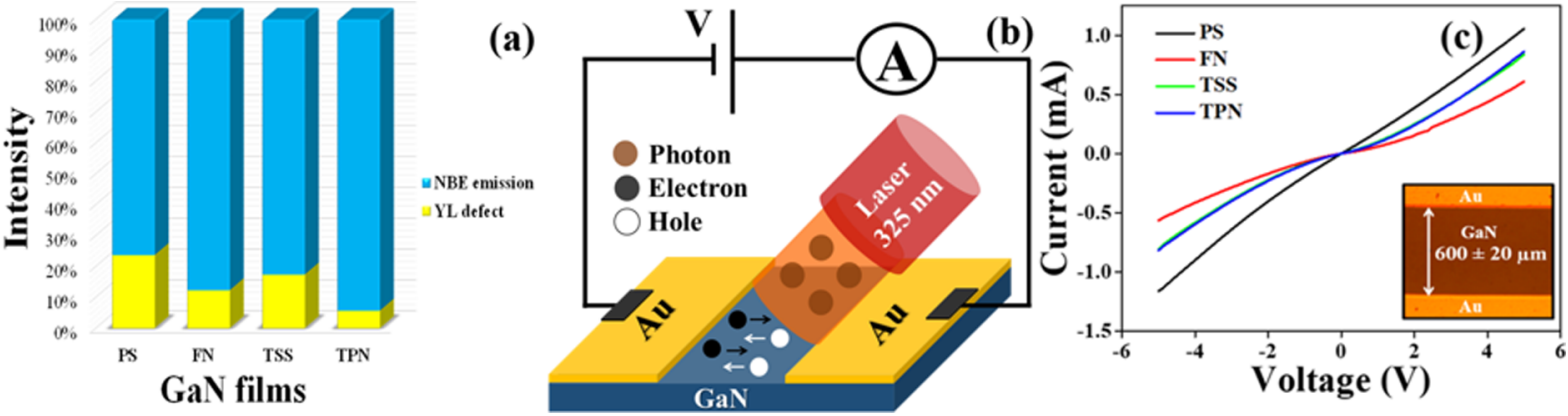

Figure 2. (a) Bar diagram representing the intensity ratio of the defect band to near-band-edge emission of GaN samples. (b) Schematic diagram representing the fabricated GaN UV PDs under $325 \mathrm{~nm}$ illumination and (c) current-voltage curves under dark conditions, with the inset displaying the optical image of the fabricated UV PDs.

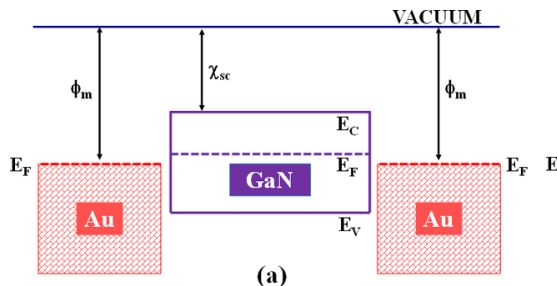

(a)

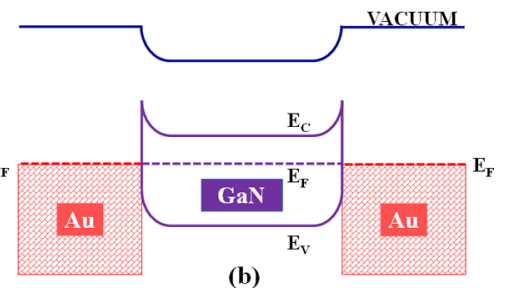

(b)

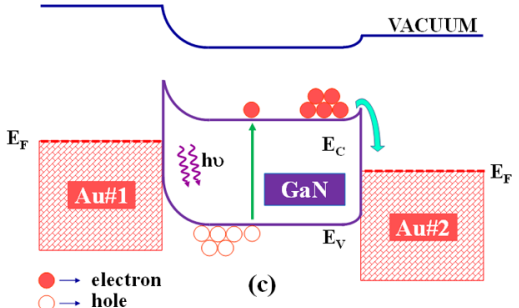

Figure 3. Schematic band diagram representing the gold metal and GaN semiconductor (a) before junction formation and (b) after junction formation in dark and (c) under light conditions along with the applied bias for the GaN UV PD.

explained based on their energetics. The favorable energetics of the surface pits (ideal sites) for oxygen chemisorption ${ }^{35}$ leads to the increment of the size along with the dissolution of gallium oxide in the solution.

The intensity ratio of the defect band $(\sim 2.1 \mathrm{eV})$ to nearband-edge emission $(\sim 3.4 \mathrm{eV})$, that is, $I_{\mathrm{DEF}} / I_{\mathrm{NBE}}$, and currentvoltage $(I-V)$ characteristics of the nanostructure-based UV PDs under dark conditions along with the schematic of the fabricated device are shown in Figure 2. Figure 2a shows the $I_{\mathrm{DEF}} / I_{\mathrm{NBE}}$ ratio as obtained from the room-temperature photoluminescence (PL) spectroscopy (Figure S2 of the Supporting Information), which confirms the suppression of the defect states in the surface-engineered GaN films. It is evident from the results that $I_{\mathrm{DEF}} / I_{\mathrm{NBE}}$ decreases from PS to FN but increases further in TSS. However, the TPN was observed to exhibit minimum defects with the least intensity of $I_{\mathrm{DEF}} / I_{\mathrm{NBE}}$. The $I-V$ curve of the pristine PS PD displayed a linear behavior, which became Schottky-type for etched PDs (Figure 2c). The dark current values for PS, FN, TSS, and TPN were in the order of $1 \mathrm{~mA}( \pm 0.1 \mathrm{~mA})$. Frequently, the thicknesses of the GaN film utilized for photodetection are of the order of 3$5 \mu \mathrm{m}$. However, the thickness of the GaN film utilized in this study was very low $\sim 600 \mathrm{~nm}$. Accordingly, the grown GaN thin film showcased a slightly higher dislocation density in the film. Such high dark current values are resulted because of the presence of more dislocations which lead to defect-assisted tunneling in the grown GaN films (PS). However, under UV illumination $\left(325 \mathrm{~nm}, 13 \mathrm{~mW} \mathrm{~cm}{ }^{-2}\right)$, enhanced photocurrent values of $18.6,78.8$, and $84.3 \mu \mathrm{A}$ for FN-, TSS-, and TPN-based UV PDs were observed. The increment in the photocurrent could be attributed to the following reasons: first, the elongated nanorod structure leads to an increased delocalization of electrons, thereby reducing the photogenerated carrier recombination. ${ }^{36,37}$ As a result, the surface-engineered GaN films displayed a threefold enhancement in photocurrent (the difference between light and dark currents) when compared with the PS. Second, elimination of surface states (vacancies, adsorbates, contaminant, etc.) is related to defects/traps that screen the effective charge transport and act as a recombination/trap center. ${ }^{38}$ Also, the charge carrier extraction was significantly improved because of the development of crystallographic planes in chemically modified samples which behave as excellent light scatterers.

Figure 3 represents a model based on the energy band theory to understand the mechanism of photocurrent conduction in fabricated UV PDs. Schematics of the energy band diagram in dark and under UV illumination are depicted in Figure $3 a-c$, respectively. Prior to the deposition of gold electrodes, all parameters such as work function of the metal and electron affinity of the semiconductor are represented in Figure 3a. In thermal equilibrium, when the metal contacts the semiconductor, alignment of Fermi levels takes place, leading to the formation of a Schottky-type metal-semiconductor (MS) contact. The Schottky barrier height was calculated to be around $1 \mathrm{eV}$ considering the work function of gold and the electron affinity of GaN. Because of symmetric contacts, the 


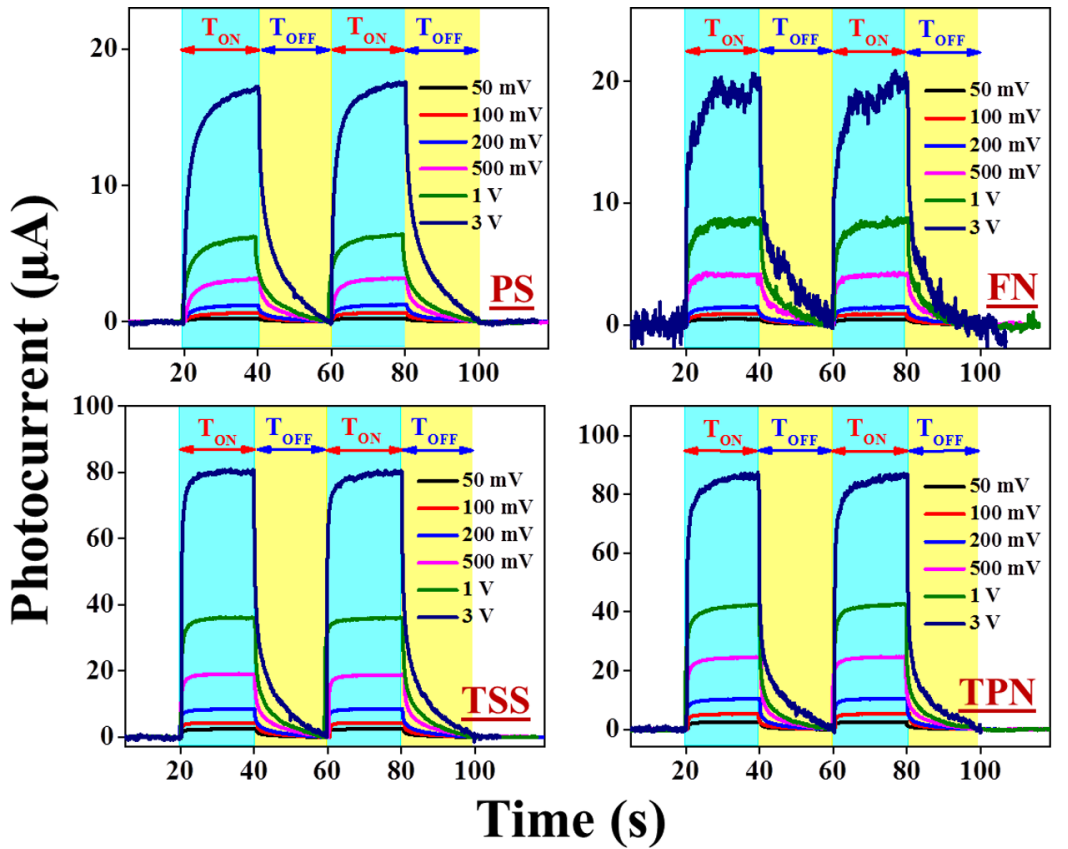

Figure 4. Time-dependent photoresponse of the fabricated UV PDs at different bias conditions.

width of the depletion region at the Au\#1-GaN interface is equal to that at the Au\#2-GaN interface (Figure 3b). Under UV illumination, generation of electron-hole pairs and transport and collection of charge carriers take place (Figure $3 c$ ). Under applied bias (contact Au\#1 is fed with negative bias and contact Au\#2 is fed with positive bias), the absorption of light near the reverse-biased junction ( $\mathrm{GaN}$ and $\mathrm{Au \# 1}$ ) creates electron-hole pairs which are separated, thus creating the photocurrent. The electrons tend to move toward Au\#2 because of the forward bias at the semiconductor, which helps in reducing the built-in potential and allows charge collection.

The time-dependent photoresponse of the fabricated UV PDs based on nanostructured GaN films by periodically switching on and off the light with a power of $13 \mathrm{~mW} \mathrm{~cm}^{-2}$, under varying bias voltage $(50 \mathrm{mV}$ to $3 \mathrm{~V})$, is depicted in Figure 4. When the light was turned on, a rise in photocurrent approaching saturation value was witnessed, which decayed after turning off the laser. The photocurrent increased with the applied bias because of the enhancement in the generation and collection of photoexcited charge carriers with increasing voltage. The fabricated PDs displayed stable and repeatable results. The responsivity of the fabricated PDs is defined as the photocurrent generated per unit power of incident light on the effective area of a photoconductor, which can be expressed as ${ }^{21}$

$$
R=\frac{\Delta I}{P A}
$$

where $\Delta I$ is the photocurrent, $P$ is the optical power density, and $A$ is the device area. The calculated responsivity values of PS, FN, TSS, and TPN GaN film-based UV PDs were 4.4, 5.6, 24.5 , and $25.9( \pm 0.3) \mathrm{mA} \mathrm{W}^{-1}$, respectively, at an applied bias of $3 \mathrm{~V}$ (Table 1$)$.

Figure 5 represents the variation in the photocurrent as well as the responsivity in the UV PD of GaN films before and after etching. An increment of $\sim 1.3,5.6$, and 5.9 times the responsivity of FN-, TSS-, and TPN-based UV PDs with respect to the pristine UV PD was observed. The sixfold increment in the responsivity of the surface-engineered $\mathrm{GaN}$
Table 1. Calculated Values of Photocurrent, Responsivity, NEP, and Detectivity of the Fabricated GaN UV PDs under $325 \mathrm{~nm}$ Illumination at a Bias of $3 \mathrm{~V}$

\begin{tabular}{lcccc} 
& $\begin{array}{c}\text { photocurrent } \\
(\mu \mathrm{A})\end{array}$ & $\begin{array}{c}\text { responsivity } \\
\left(\mathrm{mA} \mathrm{W}^{-1}\right)\end{array}$ & $\begin{array}{c}\mathrm{NEP} \\
\left(\times 10^{-10} \mathrm{~W} \mathrm{~Hz}^{-1 / 2}\right)\end{array}$ & $\begin{array}{c}\text { detectivity } \\
\left(\times 10^{8} \text { jones }\right)\end{array}$ \\
PS & 15.0 & 4.4 & 31.7 & 0.43 \\
FN & 18.6 & 5.6 & 17.5 & 0.76 \\
TSS & 78.8 & 24.5 & 4.8 & 2.73 \\
TPN & 84.3 & 25.9 & 4.7 & 2.83 \\
\hline
\end{tabular}

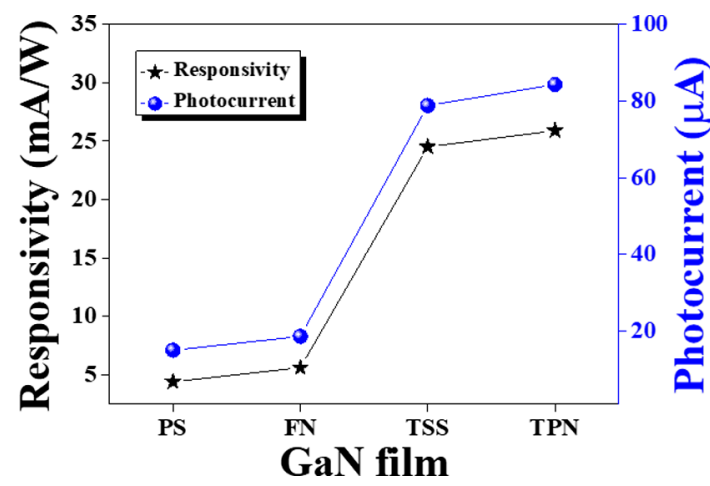

Figure 5. Graphical representation of the change in photocurrent and responsivity of the GaN UV PDs fabricated before and after etching.

UV PD is attributed to the network-structured nanorods on the chemically modified surface. It is noteworthy to mention that the material is the same in all fabricated UV PDs; hence, the observed enhancement in responsivity was attributed to either the increased photon absorption or efficient carrier transport.

According to Garrido et al., ${ }^{39}$ the photoconductive responsivity is dependent on two factors: the photogenerated free carriers $(\Delta n)$ and effective conduction cross section $(\Delta S)$. Therefore, the photoconductive gain is dominated by the modulation in photogenerated carrier concentration and effective conduction cross section. Hence, the expression for the current responsivity can be redefined as 39 


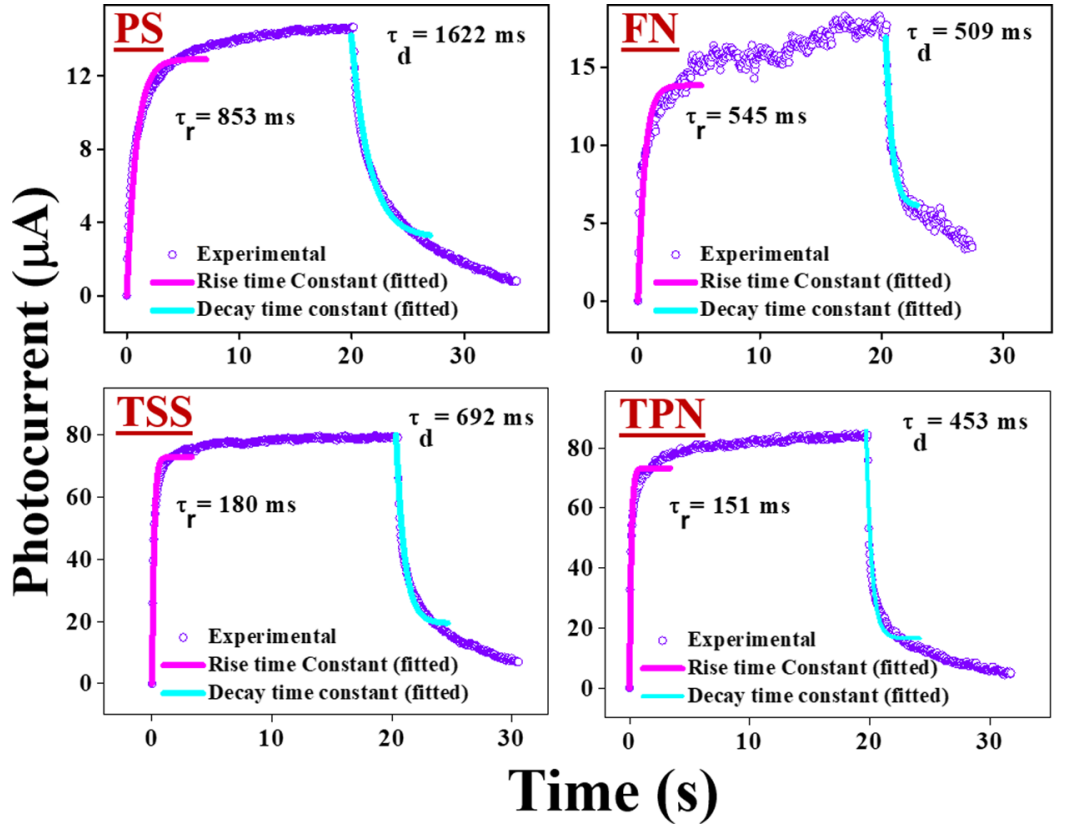

Figure 6. Rise time and decay time constant curves fitted with the experimental data obtained for PS-, FN-, TSS-, and TPN-based UV PDs.

$$
R=\frac{q V_{\mathrm{B}} \mu_{\mathrm{e}}}{L P_{\mathrm{opt}}}(S \Delta n+n \Delta S)
$$

where $q$ is the electronic charge, $V_{\mathrm{B}}$ is the bias voltage, $\mu_{\mathrm{e}}$ is the electron mobility, $L$ is the distance between the electrodes, and $P_{\text {opt }}$ is the optical power. It is evident from eq 2 that the parameters affecting responsivity (directly proportional) are photogenerated free carrier concentration and conduction cross section. The conduction cross section is significantly affected by the presence of defect states (dislocations, grain boundaries, vacancies, voids, etc.) in the films. Upon illumination of light, the defect states get charged and form a depletion region around them which creates discontinuities in the transport of photogenerated carriers and thus reduces the effective conduction cross section. Also, surface states/imperfections may also act as trapping and recombination centers and reduce the collection of carriers. It is important to mention that these defect states do not hinder the absorption of incident light, but they considerably reduce the conduction and collection of photogenerated carriers. Therefore, the enhancement in responsivity of the etched UV PDs can be attributed to the higher amount of photogenerated carriers (because of the large surface area and reduced reflection) and enhanced conduction cross section (because of the reduction in the defect/surface states).

The two key figure-of-merit parameters that determine the performance of a PD are the NEP and detectivity. The NEP is defined as the optical signal in watts at which the electrical signal-to-noise ratio in the detector is equal to unity $(0 \mathrm{~dB})$, when the bandwidth is limited to $1 \mathrm{~Hz}$ and the detectivity is described as the capability of a PD to detect the weakest light signal. The detectivity $(D)$ and NEP of the fabricated nanostructured devices are expressed by the following equation $^{40}$

$$
D=\frac{(A \Delta f)^{1 / 2}}{\mathrm{NEP}}
$$

where

$$
\mathrm{NEP}=\frac{\overline{i_{\mathrm{n}}}{ }^{1 / 2}}{R}
$$

Here, $\Delta f$ is the electrical bandwidth and ${\overline{i_{\mathrm{n}}}}^{2 / 2}$ is the measured noise. The calculated values of detectivity and NEP are displayed in Table 1 . The low NEP in the order of $10^{-10} \mathrm{~W}$ $\mathrm{Hz}^{-1 / 2}$ in surface-engineered nanostructured UV PDs indicates very low noise power in the devices, which makes them suitable candidates for highly sensitive GaN UV PDs. Accordingly, on the basis of the responsivity and dark current, the detectivity was calculated for all the fabricated surface-engineered UV PDs. The pristine PD exhibited the lowest detectivity $\left(0.43 \times 10^{8}\right.$ Jones), whereas the surface-engineered PD demonstrated nearly sixfold enhancement in detectivity, that is, $2.83 \times 10^{8}$ Jones. It indicates that surface engineering is a convenient approach for the development of efficient PDs which exhibit detectivity comparable to that of silicon-based PDs. ${ }^{40-42}$

Figure 6 shows the time-resolved photocurrent response of the device where the rise time constant (time at which the photocurrent reaches $63 \%$ of its peak value) and decay time constant (time at which the photocurrent reaches $37 \%$ from its peak value) curves which are fitted (using mathematical equations) in accordance with the experimental data from which the rise time (time at which the device response rises from 10 to $90 \%$ ) and decay time (time at which the device response decreases from 90 to $10 \%$ ) are calculated. These rise and decay time constants are fitted by the following equations ${ }^{43}$

$$
\begin{aligned}
& I(t)=I_{\mathrm{o}}\left(1-\mathrm{e}^{-t / \tau_{\mathrm{r}}}\right) \\
& I(t)=I_{\mathrm{o}}\left(\mathrm{e}^{-t / \tau_{\mathrm{d}}}\right)
\end{aligned}
$$

where $I_{\mathrm{o}}$ is the maximum current at a particular time, $\tau_{\mathrm{r}}$ is the rise time constant, $\tau_{\mathrm{d}}$ is the decay time constant, and $t$ is the response time. The fitting results exhibited the rise time of $1874,1197,395$, and $332( \pm 10) \mathrm{ms}$ and the decay time of $3564,1118,1520$, and 995 ( \pm 50$)$ ms for PS-, FN-, TSS-, and TPN-based UV PDs, respectively (as shown in Table 2). The lowest rise and decay time for TPN-based UV PDs ensure fast 
Table 2. Fitted Values of Rise and Decay Time Constants $\left(\tau_{\mathrm{r}}\right.$ and $\left.\tau_{\mathrm{d}}\right)$ and the Calculated Values of Rise and Decay Time $\left(t_{\mathrm{r}}\right.$ and $\left.t_{\mathrm{d}}\right)$ of the Fabricated UV PDs

\begin{tabular}{lcccc} 
& $\tau_{\mathrm{r}}(\mathrm{ms})$ & $\tau_{\mathrm{d}}(\mathrm{ms})$ & $t_{\mathrm{r}}(\mathrm{ms})$ & $t_{\mathrm{d}}(\mathrm{ms})$ \\
PS & 853 & 1622 & 1874 & 3564 \\
FN & 545 & 509 & 1197 & 1118 \\
TSS & 180 & 692 & 395 & 1520 \\
TPN & 151 & 453 & 332 & 995 \\
\hline
\end{tabular}

response of the fabricated UV PDs after exposure to UV radiation along with quick recovery time. It was observed that the decay time was decreased in FN $(1118 \mathrm{~ms})$, but it further increased for TSS $(1520 \mathrm{~ms})$ and ultimately the lowest value was achieved for TPN (995 ms). The higher decay time observed in TSS with respect to FN could be attributed to the higher defect states (as evident from the PL results) which might have acted as a trap center and ultimately increased the decay time. This can be explained based on morphological aspects, where FN and TPN exhibited nanorod-like structures on the surface. However, no such trend was observed in the rise time of the devices.

The enhanced responsivity observed in the surfaceengineered UV PDs (from 4.4 to $25.9 \mathrm{~mA} \mathrm{~W}^{-1}$ ) was attributed to the formation of nanorods (low-dimensional structures) which can assist in shortening the transient time of the photogenerated carriers and ultimately boost the device performance. However, it was observed that the responsivities of TSS- and TPN-based UV PDs are nearly similar, but when the response time is considered, TPN-based UV PDs have faster response time ( $332 \mathrm{~ms})$ than TSS-based UV PDs (395 $\mathrm{ms})$, which makes them superior of all the surface-engineered PDs. The decay time of surface-engineered UV PDs (995 ms) was observed to be much better when compared to that of PS (3564 ms). The increment in decay time usually occurs because of the phenomenon of persistent photoconductivity (PPC). The occurrence of PPC in GaN is an inevitable phenomenon because of the presence of defects, surface states, and oxygen contamination. ${ }^{44-46}$ The defect/surface/trap states get ionized when the incident radiation falls and act as recombination centers for photogenerated carriers after the light is switched off. $^{47}$ The efficiency of these trap states decreases with time (few milliseconds), which leads to PPC and increases the decay time of devices. Hence, the decrease in decay time of the chemically engineered UV PDs indicates reduction in defect states in the nanostructured films. Also, the response time (both on rise and decay) of the fabricated UV PDs is better than most of the previously reported low-dimensional nanostructured PDs, ranging from seconds to tens of minutes or even hours, which is highlighted in Table $3 .^{48-55}$ Therefore, by utilizing these surface-engineered structures, the response time and recovery speed of MSM-based GaN UV PDs can be dramatically reduced, which makes them ideal for the faster switching applications.

\section{CONCLUSIONS}

Surface-engineered nanostructure-based nonpolar MSM-GaN high-performance PDs are demonstrated for the detection of UV A radiation. Transformation from PS to FN and TSS was observed after a three-step chemical etching procedure. The chemically engineered UV PDs displayed significant suppression of defect states, which ultimately improved the photoresponse and enhanced the device performance. The triangular
Table 3. Comparative Study of Rise Time and Decay Time of Various UV PDs

\begin{tabular}{llll}
\multicolumn{1}{c}{ photodetector structure } & $\begin{array}{c}\text { rise time } \\
(\mathrm{ms})\end{array}$ & $\begin{array}{c}\text { fall time } \\
(\mathrm{ms})\end{array}$ & \multicolumn{1}{c}{ refs } \\
GaN nanostructures & 332 & 992 & this work \\
$\mathrm{ZnO}$ nanoflowers/graphene & 1000 & 6700 & 48 \\
$\mathrm{ZnO}$ nanoflowers/ $\mathrm{MoS}_{2}$ & 6100 & 9000 & 48 \\
$\mathrm{BiSnO}_{2}$ & 3000 & 1000 & 49 \\
$\mathrm{p}-\mathrm{CNT} / \mathrm{ZnO}$ & 1400 & 2300 & 50 \\
$\mathrm{p}-\mathrm{Si} / \mathrm{n}-\mathrm{ZnO}$ nanotubes & 440 & 599 & 51 \\
bicrystalline $\mathrm{GaN}$ nanowire & 144 & 256 & 52 \\
$\mathrm{ZnO}$ nanocrystals & $<0.5$ & & 53 \\
graphene- $\beta$-Ga $\mathrm{O}_{3}$ & 9483 & 21919 & 54 \\
graphene quantum & 3700 & & 55 \\
dots/graphene & & & \\
\hline
\end{tabular}

nanorod-based TPN PDs displayed a responsivity of $25.9 \mathrm{~mA}$ $\mathrm{W}^{-1}$ compared with $4.4 \mathrm{~mA} \mathrm{~W}^{-1}$ for PDs fabricated on the pristine film. The detectivity of TPN PDs revealed a drastic increment of $\sim 6.6$-fold, while the NEP was reduced by $\sim 6.7$ times. The nanorod-based PDs ensured fast switching by reducing the response time to $332 \mathrm{~ms}$ from $1874 \mathrm{~ms}$. These enhancements occurred via increased light absorption and higher conduction cross section in the developed nanostructures. We conclude that surface-engineering-based nanostructured MSM-GaN PD is a promising strategy to fabricate GaN-based efficient PDs.

\section{EXPERIMENTAL SECTION}

The epitaxial a-plane GaN film was grown on an $r$-plane sapphire substrate by a plasma-assisted MBE (PAMBE, Riber Compact 21) system equipped with standard Knudsen cells and an $\mathrm{ADDON}$ radio frequency (rf) plasma source to supply active nitrogen species. Prior to loading the substrate in the load-lock chamber, the $r$-plane sapphire substrate was precleaned chemically by degreasing in acetone for 2-3 min, followed by etching in $1: 3 \mathrm{H}_{3} \mathrm{PO}_{4} / \mathrm{H}_{2} \mathrm{SO}_{4}$ solution at $60{ }^{\circ} \mathrm{C}$ for $5 \mathrm{~min}$ and further blowing with dry nitrogen gas. In situ thermal annealing was carried out at $600{ }^{\circ} \mathrm{C}$ in the buffer chamber to remove the residual contaminants from the substrate. Nitridation of the substrate was performed at a low substrate temperature $\left(450^{\circ} \mathrm{C}\right)$. A low-temperature $\mathrm{GaN}$ buffer layer was deposited at $530{ }^{\circ} \mathrm{C}$ under Ga-rich conditions. The growth of $\mathrm{GaN}$ was performed at $730{ }^{\circ} \mathrm{C}$ with a gallium beam equivalent pressure of $1.0 \times 10^{-6}$ Torr and an rf power of $500 \mathrm{~W}$. After the epitaxial growth of the a-plane $\mathrm{GaN}$ film on the $r$-plane sapphire substrate, post-annealing for $27 \mathrm{~min}$ was performed in nitrogen plasma under ambient conditions to convert the remaining adsorbed $\mathrm{Ga}$ adatoms into the $\mathrm{GaN}$ film. The pristine nonpolar GaN film was initially etched with $\mathrm{KOH}$ solution and periodically immersed in $\mathrm{H}_{3} \mathrm{PO}_{4}$ and $\mathrm{KOH}$ solutions afterward (as shown in Figure 1). The temperature of the solutions and etching time were kept constant at $80{ }^{\circ} \mathrm{C}$ and $10 \mathrm{~min}$, respectively. The etched films were then rinsed in distilled water and dried by pressurized nitrogen gas. The samples in this article are abbreviated as pristine pyramidal structure (PS), flat nanorods (FN), trigonal shape structure (TSS), and trigonal prism shaped nanorods (TPN), as witnessed from the observed surface morphology. MSM structure-based UV PDs were fabricated by depositing gold contacts (thickness $\approx 150 \pm 5 \mathrm{~nm}$ and separation $\approx 600 \pm 20$ $\mu \mathrm{m})$ onto the film surface using the PVD technique. The 
morphology of the samples was examined by Supra 40VP FESEM (Zeiss Germany). Photodetection measurements were performed on a semiconductor parameter analyzer connected to a probe station setup (Cascade Microtech EPS150TRIAX), which has shield enclosure (EPS-ACC-SE750) for low-signal measurements. The spectrometer was equipped with a focused laser $\left(\lambda=325 \mathrm{~nm}\right.$, power density $\left.=13 \mathrm{~mW} \mathrm{~cm}^{-2}\right)$ for the generation and detection of UV A radiation.

\section{ASSOCIATED CONTENT}

\section{S Supporting Information}

The Supporting Information is available free of charge on the ACS Publications website at DOI: 10.1021/acsomega.7b02024.

AFM and PL results of the samples (PDF)

\section{AUTHOR INFORMATION}

\section{Corresponding Author}

*E-mail: govind@nplindia.org, govindnpl@gmail.com. Phone: +91-1145608403 (G.G.).

\section{ORCID}

Govind Gupta: 0000-0001-5006-7623

\section{Notes}

The authors declare no competing financial interest.

\section{ACKNOWLEDGMENTS}

The authors acknowledge the Director, CSIR-NPL India, for his constant support. The work is financially supported by the Department of Science and Technology (Govt. of India) under grant aid DST/TM/CERI/C245(G). The authors acknowledge Pooja Singh for contact fabrication. The authors also acknowledge Dr. Ritu Srivatsava for AFM measurements. Shibin Krishna thanks DST (GoI) and SIMCO Global Tech. \& System Ltd for financial support under the prestigious Prime Minister Doctoral Fellowship scheme.

\section{REFERENCES}

(1) Huang, M. H.; Mao, S.; Feick, H.; Yan, H.; Wu, Y.; Kind, H.; Weber, E.; Russo, R.; Yang, P. Room Temperature Ultraviolet Nanowires Nanolasers. Science 2001, 292, 1897-1899.

(2) Wang, F.; Zhao, D.; Guo, Z.; Liu, L.; Zhang, Z.; Shen, D. Artificial leaf structures as a UV detector formed by the self-assembly of $\mathrm{ZnO}$ nanoparticles. Nanoscale 2013, 5, 2864-2869.

(3) Zhang, X.; Liu, B.; Liu, Q.; Yang, W.; Xiong, C.; Li, J.; Jiang, X. Ultrasensitive and Highly Selective Photodetections of UV-A Rays Based on Individual Bicrystalline GaN Nanowire. ACS Appl. Mater. Interfaces 2017, 9, 2669-2677.

(4) Walker, D.; Razeghi, M. The Development of Nitride based UV Photodetectors. Opto-Electron. Rev. 2000, 8, 25-42.

(5) Ruterana, P.; Albrecht, M.; Neugebauer, J. Nitride Semiconductors: Handbook on Materials and Devices; Wiley Publications, 2006.

(6) Alaie, Z.; Nejad, S. M.; Yousefi, M. H. Recent Advances in Ultraviolet Photodetectors. Mater. Sci. Semicond. Process. 2015, 29, 1655.

(7) Bernardini, F.; Fiorentini, V. Spontaneous versus Piezoelectric Polarization in III-V Nitrides: Conceptual Aspects and Practical Consequences. Phys. Status Solidi B 1999, 216, 391-398.

(8) Mishra, M.; Krishna, T. C. S.; Aggarwal, N.; Gupta, G. Surface Chemistry and Electronic Structure of Nonpolar and Polar GaN Films. Appl. Surf. Sci. 2015, 345, 440-447.

(9) Mishra, M.; Krishna, T. C. S.; Aggarwal, N.; Vihari, S.; Gupta, G. Electronic Structure Analysis of GaN Films Grown on r- and a-plane Sapphire. J. Alloys Compd. 2015, 645, 230-234.

(10) Farrell, R. M.; Young, E. C.; Wu, F.; DenBaars, S. P.; Speck, J. S. Materials and Growth Issue for High-Performance Nonpolar and
Semipolar Light-Emitting Diodes. Semicond. Sci. Technol. 2012, 27, 024001-024014.

(11) Waltereit, P.; Brandt, O.; Trampert, A.; Grahn, H. T.; Menniger, J.; Ramsteiner, M.; Reiche, M.; Ploog, K. H. Nitride Semiconductors Free of Electrostatic Fields for Efficient White Light Emitting Diodes. Nature 2000, 406, 865-868.

(12) Mukundan, S.; Roul, B.; Shetty, A.; Chandan, G.; Mohan, L.; Krupanidhi, S. B. Enhanced UV Detection by Nonpolar Epitaxial GaN Films. AIP Adv. 2015, 5, 127208-127214.

(13) Gundimeda, A.; Krishna, S.; Aggarwal, N.; Sharma, A.; Sharma, N. D.; Maurya, K. K.; Husale, S.; Gupta, G. Fabrication of Nonpolar GaN based Highly Responsive and Fast UV Photodetector. Appl. Phys. Lett. 2017, 110, 103507.

(14) Konstantatos, G.; Sargent, E. H. Nanostructured Materials for Photon Detection. Nat. Nanotechnol. 2010, 5, 391-400.

(15) Chen, H.; Liu, H.; Zhang, Z.; Hu, K.; Fang, X. Nanostructured Photodetectors: From Ultraviolet to Terahertz. Adv. Mater. 2016, 28, 403-433.

(16) Peng, L.; Hu, L.; Fang, X. Low-Dimensional Nanostructure Ultraviolet Photodetectors. Adv. Mater. 2013, 25, 5321-5328.

(17) Liu, S.; Ye, J.; Cao, Y.; Shen, Q.; Liu, Z.; Qi, L.; Guo, X. Tunable Hybrid Photodetectors with Superhigh Responsivity. Small 2009, 5, 2371-2376.

(18) Li, L.; Wu, P.; Fang, X.; Zhai, T.; Dai, L.; Liao, M.; Koide, Y.; Wang, H.; Bando, Y.; Golberg, D. Single-Crystalline CdS Nanobelts for Excellent Field-Emitters and Ultrahigh Quantum-Efficiency Photodetectors. Adv. Mater. 2010, 22, 3161-3165.

(19) Sai, H.; Kanamori, Y.; Arafune, K.; Ohshita, Y.; Yamaguchi, M. Light Trapping Effect of Submicron Surface Textures in Crystalline Si Solar Cells. Prog. Photovoltaics 2007, 15, 415-423.

(20) Lanz, T.; Lapagna, K.; Altazin, S.; Boccard, M.; Haug, F.-J.; Ballif, C.; Ruhstaller, B. Light Trapping in Solar Cells: Numerical Modelling with Measured Surface Textures. Opt. Express 2015, 23, A539-A546.

(21) Anguita, J. V.; Ahmad, M.; Haq, S.; Allam, J.; Silva, S. R. P. Ultra-broadband Light Trapping Using Nanostructured Decoupled Graphene Multilayers. Sci. Adv. 2016, 2, 1501238.

(22) Ottone, C.; Rivera, V. F.; Fontana, M.; Bejtka, K.; Onida, B.; Cauda, V. Ultralong and Mesoporous $\mathrm{ZnO}$ and $\gamma$-Al2O3 Oriented Nanowires Obtained by Template-assisted Hydrothermal Approach. J. Mater. Sci. Technol. 2014, 30, 1167-1173.

(23) Ottone, C.; Bejtka, K.; Chiodoni, A.; Farías, V.; Roppolo, I.; Canavese, G.; Stassi, S.; Cauda, V. Comprehensive Study of Templating Effect on the $\mathrm{ZnO}$ Nanostructure Formation Within Porous Hard Membrane. New J. Chem. 2014, 38, 2058-2065.

(24) Laurenti, M.; Stassi, S.; Lorenzoni, M.; Fontana, M.; Canavese, G.; Cauda, V.; Pirri, C. F. Evaluation of the Piezoelectric Properties and Voltage Generation of Flexible Zinc Oxide Thin Films. Nanotechnology 2015, 26, 215704.

(25) Podrezova, L. V.; Porro, S.; Cauda, V.; Fontana, M.; Cicero, G. Comparison between $\mathrm{ZnO}$ Nanowires grown by Chemical Vapor Deposition and Hydrothermal Synthesis. Appl. Phys. A: Mater. Sci. Process. 2013, 113, 623-632.

(26) Zhu, Z.; Chen, T.-L.; Gu, Y.; Warren, J.; Osgood, R. M. Zinc Oxide Nanowires Grown by Vapor-Phase Transport Using Selected Metal Catalysts: A Comparative Study. Chem. Mater. 2005, 17, 42274234.

(27) Kumar, M.; Bhat, T. N.; Rajpalke, M. K.; Roul, B.; Misra, P.; Kukreja, L. M.; Sinha, N.; Kalghatgi, A. T.; Krupanidhi, S. B. Selfassembled Flower-like Nanostructures of $\mathrm{InN}$ and GaN Grown by Plasma-Assisted Molecular Beam Epitaxy. Bull. Mater. Sci. 2010, 33, 221-226.

(28) Aggarwal, N.; Krishna, S.; Sharma, A.; Goswami, L.; Kumar, D.; Husale, S.; Gupta, G. A highly Responsive Self-driven UV Photodetector using GaN Nanoflowers. Adv. Electr. Mater. 2017, 3, 1700036.

(29) Laurenti, M.; Stassi, S.; Canavese, G.; Cauda, V. Surface Engineering of Nanostructured $\mathrm{ZnO}$ Surfaces. Adv. Mater. Interfaces 2017, 4, 1600758. 
(30) Kung, S.-C.; Van der Veer, W. E.; Yang, F.; Donavan, K. C.; Penner, R. M. $20 \mu$ s Photocurrent Response from Lithographically Patterned Nanocrystalline Cadmium Selenide Nanowires. Nano Lett. 2010, 10, 1481-1485.

(31) Jung, S.; Song, K.-R.; Lee, S.-N.; Kim, H. Wet Chemical Etching of Semipolar GaN Planes to Obtain Brighter and Cost-Competitive Light Emitters. Adv. Mater. 2013, 25, 4470-4476.

(32) Zhuang, D.; Edgar, J. H. Wet Etching of GaN, AlN, and SiC: A Review. Mater. Sci. Eng. R Rep. 2005, 48, 1-46.

(33) Tamboli, A. C.; Hirai, A.; Nakamura, S.; DenBaars, S. P.; Hu, E. L. Photoelectrochemical Etching of p-type GaN Heterostructures. Appl. Phys. Lett. 2009, 94, 151113.

(34) Hardy, M. T.; Kelchner, K. M.; Lin, Y.-D.; Hsu, P. S.; Fujito, K.; Ohta, H.; Speck, J. S.; Nakamura, S.; DenBaars, S. P. m-Plane GaNBased Blue Superluminescent Diodes Fabricated Using Selective Chemical Wet Etching. Appl. Phys. Exp. 2009, 2, 121004.

(35) Mishra, M.; Krishna, T. C. S.; Aggarwal, N.; Kaur, M.; Singh, S.; Gupta, G. Pit Assisted Oxygen Chemisorption of GaN Surfaces. Phys. Chem. Chem. Phys. 2015, 17, 15201-15208.

(36) Cansizoglu, H.; Cansizoglu, M. F.; Watanabe, F.; Karabacak, T. Enhanced Photocurrent and Dynamic Response in Vertically Aligned In2S3/Ag Core/Shell Nanorod Array Photoconductive Devices. ACS Appl. Mater. Interfaces 2014, 6, 8673-8682.

(37) Leelavathi, A.; Madras, G.; Ravishankar, N. Origin of Enhanced Photocatalytic Activity and Photoconduction in High Aspect Ratio ZnO Nanorods. Phys. Chem. Chem. Phys. 2013, 15, 10795-10802.

(38) Mishra, M.; Gundimeda, A.; Krishna, S.; Aggarwal, N.; Gahtori, B.; Dilawar, N.; Aggarwal, V. V.; Singh, M.; Rakshit, R.; Gupta, G. Wet chemical etching induced stress relaxed nanostructures on polar \& non-polar epitaxial GaN films. Phys. Chem. Chem. Phys. 2017, 19, 8787-8801.

(39) Garrido, J. A.; Monroy, E.; Izpura, I.; Muñoz, E. Photoconductive Gain Modelling of GaN Photodetectors. Semicond. Sci. Technol. 1998, 13, 563-568.

(40) Guo, F.; Yang, B.; Yuan, Y.; Xiao, Z.; Dong, Q.; Bi, Y.; Huang, J. A Nanocomposite Ultraviolet Photodetector based on Interfacial Trapcontrolled Charge Injection,. Nat. Nanotechnol. 2012, 7, 798-802.

(41) Qi, J.; Zhou, X.; Yang, D.; Qiao, W.; Ma, D.; Wang, Z. Y. Optimization of Solubility, Film Morphology and Photodetector Performance by Molecular Side-Chain Engineering of Low-Bandgap Thienothiadiazole-Based Polymers. Adv. Funct. Mater. 2014, 24, $7605-7612$.

(42) Gong, X.; Tong, M.; Xia, Y.; Cai, W.; Moon, J. S.; Cao, Y.; Yu, G.; Shieh, C.-L.; Nilsson, B.; Heeger, A. J. High-Detectivity Polymer Photodetectors with Spectral Response from $300 \mathrm{~nm}$ to $1450 \mathrm{~nm}$. Science 2009, 325, 1665-1667.

(43) Jin, Z.; Gao, L.; Zhou, Q.; Wang, J. High-performance Flexible Ultraviolet Photoconductors based on Solution-processed Ultrathin $\mathrm{ZnO} / \mathrm{Au}$ Nanoparticle Composite Films. Sci. Rep. 2014, 4, 4268.

(44) Polyakov, A. Y.; Smirnov, N. B.; Govorkov, A. V.; Mil'vidskii, M. G.; Redwing, J. M.; Shin, M.; Skowronski, M.; Greve, D. W.; Wilson, R. G. Properties of Si Donors and Persistent Photoconductivity in AlGaN. Solid State Electron. 1998, 42, 627-635.

(45) Qiu, C. H.; Pankove, J. I. Deep Levels and Persistent Photoconductivity in GaN Thin Films. Appl. Phys. Lett. 1997, 70, 1983-1985.

(46) Reddy, C. V.; Balakrishnan, K.; Okumura, H.; Yoshida, S. The Origin of Persistent Photoconductivity and its Relationship with Yellow Luminescence in Molecular Beam Epitaxy grown Undoped GaN. Appl. Phys. Lett. 1998, 73, 244-246.

(47) Binet, F.; Duboz, J. Y.; Rosencher, E.; Scholz, F.; Härle, V. Mechanisms of Recombination in GaN Photodetectors. Appl. Phys. Lett. 1996, 69, 1202-1204.

(48) Lee, Y. B.; Kim, S. K.; Lim, Y. R.; Jeon, I. S.; Song, W.; Myung, S.; Lee, S. S.; Lim, J.; An, K.-S. Dimensional-Hybrid Structures of 2D Materials with $\mathrm{ZnO}$ Nanostructures via $\mathrm{pH}$-Mediated Hydrothermal Growth for Flexible UV Photodetectors. ACS Appl. Mater. Interfaces 2017, 9, 15031-15037.
(49) Pan, S.; Liu, Q.; Zhao, J.; Li, G. Ultrahigh Detectivity and Wide Dynamic Range Ultraviolet Photodetectors Based on $\mathrm{Bi}_{\mathrm{x}} \mathrm{Sn}_{1-\mathrm{x}} \mathrm{O}_{2}$ Intermediate Band Semiconductor. ACS Appl. Mater. Interfaces 2017, 9, 28737-28742.

(50) Li, G.; Suja, M.; Chen, M.; Bekyarova, E.; Haddon, R. C.; Liu, J.; Itkis, M. E. Visible-Blind UV Photodetector Based on Single-Walled Carbon Nanotube Thin Film/ZnO Vertical Heterostructures. ACS Appl. Mater. Interfaces 2017, 9, 37094-37104.

(51) Flemban, T. H.; Haque, M. A.; Ajia, I.; Alwadai, N.; Mitra, S.; Wu, T.; Roqan, I. S. A Photodetector Based on $\mathrm{p}-\mathrm{Si} / \mathrm{n}-\mathrm{ZnO}$ Nanotube Heterojunctions with High Ultraviolet Responsivity,. ACS Appl. Mater. Interfaces 2017, 9, 37120-37127.

(52) Zhang, X.; Liu, B.; Liu, Q.; Yang, W.; Xiong, C.; Li, J.; Jiang, X. Ultrasensitive and Highly Selective Photodetections of UV-A Rays Based on Individual Bicrystalline GaN Nanowire. ACS Appl. Mater. Interfaces 2017, 9, 2669-2677.

(53) Ibrahem, M. A.; Verrelli, E.; Lai, K. T.; Kyriakou, G.; Lee, A. F.; Isaacs, M. A.; Cheng, F.; O'Neill, M. Dual Wavelength (Ultraviolet and Green) Photodetectors Using Solution Processed Zinc Oxide Nanoparticles. ACS Appl. Mater. Interfaces 2017, 9, 36971-36979.

(54) Kong, W.-Y.; Wu, G.-A.; Wang, K.-Y.; Zhang, T.-F.; Zou, Y.-F.; Wang, D.-D.; Luo, L.-B. Graphene- $\beta-\mathrm{Ga}_{2} \mathrm{O}_{3}$ Heterojunction for Highly Sensitive Deep UV Photodetector Application. Adv. Mater. 2016, 28, 10725-10731.

(55) Tetsuka, H. 2D/0D graphene hybrids for visible-blind flexible UV photodetectors. Sci. Rep. 2017, 7, 5544. 